\title{
The value of differently managed cacao plantations for forest bird conservation in Sulawesi, Indonesia
}

\author{
STEFAN ABRAHAMCZYK, MICHAEL KESSLER, DADANG DWI PUTRA, \\ MATTHIAS WALTERT and TEJA TSCHARNTKE
}

\begin{abstract}
Summary
In order to assess the potential value of differently managed cacao plantations for bird conservation on Sulawesi, we surveyed birds in near-primary forest (with limited timber and rattan extraction, and some hunting), cacao plantations with remnant forest trees and plantations lacking forest trees, from February to April 2007. A total of $1650 \times 50$ m plots were visited twice and records of 87 species were obtained. Bird species richness and the number of endemics and forest specialists decreased along this gradient of forest conversion, with $20 \%$ of the forest specialists, among them ro endemics, exclusively found in forest. Species composition changed dramatically between habitat types. Sørensen indices showed a similarity of species composition between forests and plantations of $45-60 \%$ for forest specialists and $65-71 \%$ for all species. The most important environmental variable for the diversity and composition of birds was the number of remnant rainforest trees present in the plantations. Our results suggest that large, undisturbed rainforest are most important for the conservation of forest specialists and endemics but that cacao plantations, if managed to maintain a high and diverse cover of forest trees, can harbour up to $60 \%$ of forest specialists and endemics.
\end{abstract}

\section{Introduction}

Worldwide, intensive logging of primary forests and the associated habitat loss and fragmentation is the most important threat for many bird species (Bryant et al. 1997). In the humid tropics, Southeast Asia has one of the highest rates of deforestation (Achard et al. 2002). This is especially true for lowland areas in Indonesia (Thiollay 1995), and in the last decades lowland forests on islands such as Sulawesi have been strongly reduced by human activities (Waltert et al. 2004a, 2005b). Much of the logged forests have been converted into agricultural land, including both annual crops and perennial plantations of mainly oil palms, copra, coffee, and cacao. In the last 30 years, cacao has become the most important perennial crop in Sulawesi, providing cash income for millions of households (Steffan-Dewenter et al. 2007). On Sulawesi, cacao is mainly cultivated in small plantations covering only a few hectares each. Young cacao plants need taller shade trees for optimal growth conditions, and adult trees also benefit by reduced vulnerability to drought and diseases (Rice \& Greenberg 2000). Shade trees can be remnant rainforest trees or planted individuals which often have an additional economic impact. Some farmers plant fruit trees whereas others use fast growing exotics such as Gliricidia sepium (Leguminosae) to provide shade in newly established plantations previously lacking large trees. These heterogeneous management practices generate a mosaic of differently structured cacao plantations (Fox et al. 2000). 
Agroforests with cacao or coffee are often considered to be important alternative habitats for many rainforest species (Rice \& Greenberg 2000, Schroth et al. 2004, Faria et al. 2007). In many agricultural landscapes, such agroforests provide the only habitat with considerable tree cover, relatively high tree species diversity and complex structure, and provide many ecosystem functions and processes also found in primary forests (Siebert 2002, Schroth et al. 2004, SteffanDewenter et al. 2007). These plantations provide habitats and dispersal pathways and assist in the conservation of the local species composition (Faria et al. 2007). Studies that have mostly been conducted in Latin America demonstrate that traditional agroforest systems, with mixed tree species and a complex vegetation structure can support a high number of bird species, including many forest species (Greenberg et al. 1997, 2000) because this plantation type is often structurally relatively similar to secondary forests (Thiollay 1995). Thus agroforests potentially play a major role in bird conservation, but the value of agroforests for bird conservation varies largely within different types of plantations and characters of shade trees. Attributes like tree species composition, tree structure or distance to the next primary forest determine whether plantations are a suitable habitat for birds or just a permeable matrix of inferior sites that mitigate the effects of deforestation and fragmentation (Greenberg et al. 2000, Waltert et al. 2005a, Faria et al. 2006, 2007).

The Wallacea region of eastern Indonesia is one of the world's biodiversity and endemism hotspots (Stattersfield et al. 1998, Myers et al. 2000). The major island Sulawesi is home to 224 resident land bird species including eleven endemic genera and 91 (43\%) endemic species on the main island and its satellite islands (Coates et al. 1997). Most of these species are forest inhabitants and only a small number occur mainly in wetlands and grasslands. Accordingly, many endemic forest species are strongly affected by deforestation. Brook et al. (2003) predicted a loss of up to $42 \%$ of the biodiversity in Southeast Asia by 2100 if current deforestation rates continue. For Sulawesi, with its unique composition of bird communities and its high number of endemics, there are just a few studies on the impact of variable land use systems on bird diversity (Siebert 2002, Waltert et al. 2004b, Sodhi et al. 2005). None of these studies distinguish between different types of plantations with respect to the density, diversity, and structure of the shade tree cover. Furthermore, all previous studies were conducted in montane or upper submontane areas between $1,000 \mathrm{~m}$ and $1,200 \mathrm{~m}$ while there is a marked change in species composition from lowland to montane species around 1,200 m elevation (Waltert et al. 2004b). Thus, the impact of different structural characteristics of cacao plantations at lower elevations on Sulawesi is still unknown.

In our study we focus on the value of differently managed agroforest systems in contrast to near-primary forest at $800-1,150 \mathrm{~m}$, for bird species diversity and conservation in Central Sulawesi, Indonesia. The main questions are:

1. Which factor is most important for the bird diversity and the number of forest specialists and endemics?

2. What value do the different habitat types have for the conservation of forest specialists and endemics?

3. Which traits (habitat specialisation, feeding ecology, etc.) render bird species more susceptible to habitat destruction in Sulawesi?

\section{Methods}

\section{Study sites}

Our study took place in Toro Valley, about $100 \mathrm{~km}$ south of Palu, the capital city of Central Sulawesi, between February and April 2007. The valley is located on the western border of Lore Lindu National Park. This conservation area, one of the most important in the Wallacea region (Myers et al. 2000, Schroth et al. 2004), was established in 1977 and has a size of 217,991 hectares. Ninety percent of the park is covered by submontane and montane tropical forest, and 
only $10 \%$ consists of lowland forest (The Nature Conservancy 2002). Toro valley (119 $90^{\prime}-$ $120^{\circ} 16^{\prime} \mathrm{E}$ and $1^{\circ} \mathrm{O}^{\prime}-1^{\circ} \mathrm{O} 8^{\prime} \mathrm{S}$ ) is located at about $800 \mathrm{~m}$ elevation and is surrounded by mountains up to about $1,200-1,300 \mathrm{~m}$ in elevation. Mean annual temperature in the area is about $24^{\circ} \mathrm{C}$ and mean annual precipitation is about $1,750 \mathrm{~mm}$ without clear seasonal fluctuations (Bos et al. 2007). Our study was conducted in 16 plots of $50 \times 50 \mathrm{~m}$ each, four in near-primary forest and twelve in cacao plantations with different structure and composition of shade trees (Steffan-Dewenter et al. 2007). The term near-primary forest is used instead of primary forest because the forest at the margins of Lore Lindu National Park is affected by small scale disturbance by man (rattan and some timber extraction as well as subsistence hunting). The forest plots were located inside the national park at 950-1,130 m elevation. All plantations were situated outside the park but not more than $300 \mathrm{~m}$ away from the next forest border, at $800-950 \mathrm{~m}$. Data on shade tree diversity and structure were obtained from Gradstein et al. (2007) whereas distance of each plot to the closest forest margin was extracted from satellite images by Dr. S. Erasmi, Geographical Institute of the University of Göttingen, Germany.

\section{Field sampling}

Each plot was visited twice from o5h3o to 1oh3o. Birds were recorded visually and acoustically, and by systematic tape recordings (Parker 1991). For every species we recorded the minimum number of individuals present simultaneously in the plot. Species flying only above the canopy such as swifts (Apodidae) and raptors were excluded from the analysis. For taxonomy we followed Coates et al. (1997). For each plot we estimated shade tree canopy cover, number of rainforest trees, and number of planted shade trees.

\section{Data analysis}

To detect groups of study plots with similar bird community composition, we used non-metric multidimensional scaling (NMDS) based on the number of individuals of the recorded bird species with the quantitative Sörensen similarity (Bray-Curtis) index, untransformed abundance data, initially 6 axes, and 250 reiterations, as implemented in PCOrd 5.0 (Figure 1). Further NMDS analyses were performed with log-transformed abundance data, presence-absence-data, and different starting configurations; these yielded qualitatively comparable results and are not further reported here. We then used ANOVAs to detect structural differences (elevation, distance to forest, canopy cover, number of rainforest trees, number of planted shade trees, number of cacao trees) between the plot categories recovered by the NMDS analysis.

After generating the habitat types we categorised all recorded bird species into forest specialists and generalists. Forest species were those among which $>50 \%$ of all recorded individuals were found in forests, generalists those with $<50 \%$ of records in forests. Species recorded only one or two times in our data set were classified according to the literature (Coates et al. 1997). In the last step, we combined the categorised species with data of their distribution and habitat preferences to find out which bird species are of conservation interest. We estimated all species which are classified as threatened by the IUCN, the endemics and the forest specialists as potentially of conservation concern. To assess the similarity in bird species composition of the habitat types, we used the quantitative Sörensen similarity (Bray-Curtis) index and considered absolute species number as well as the number of endemics, non-endemics, generalists, and forest specialists.

Species information on distribution, stratum preferences, and diet was obtained from Coates et al. (1997). Bird species were categorized into broad feeding guilds ( $\mathrm{C}=$ carnivores, $\mathrm{F}=$ frugivores, $\mathrm{G}=$ granivores, $\mathrm{I}=$ insectivores, $\mathrm{N}=$ nectarivores, $\mathrm{O}=$ omnivores) and main strata used ( $\mathrm{G}=$ ground \& understorey, $\mathrm{M}=$ midstorey, $\mathrm{C}=$ canopy). Data on mean body weight of the bird species were taken, as far as available, from Del Hoyo et al. (1992-2006). Additionally we used Feare \& Craig (1999), Maher (1991), Higgins \& Peter (2002), Higgins et al. (2006) and 


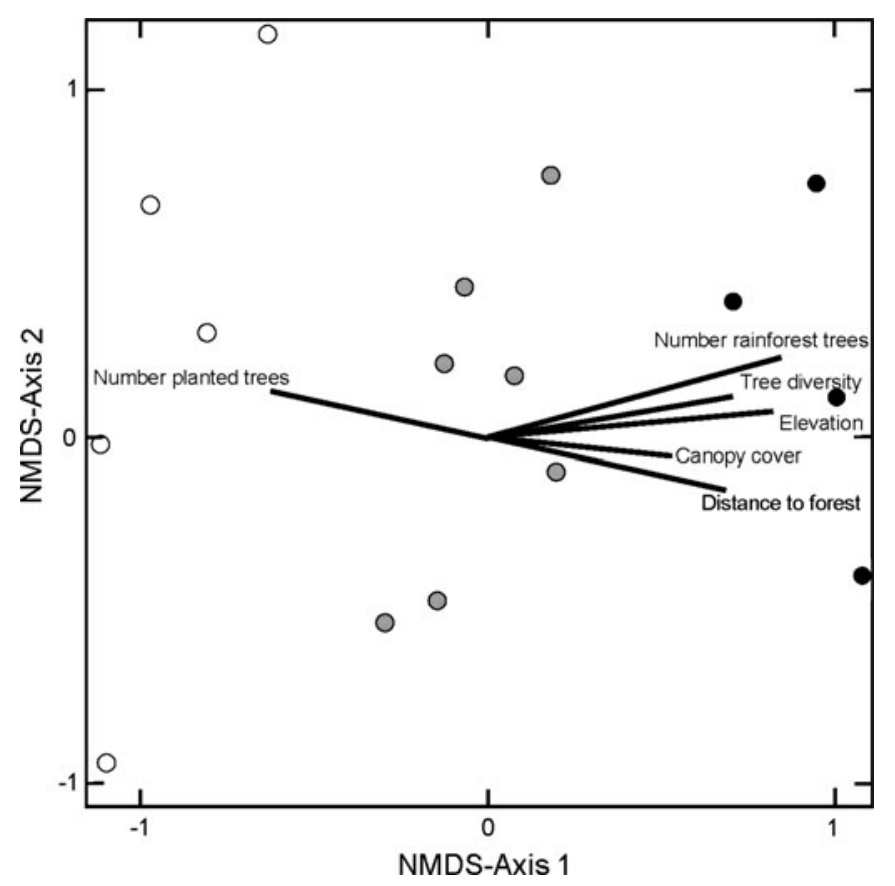

Figure 1. Results of the NMDS ordination analysis based on bird community composition, showing the differentiation into the three habitat categories near-primary forest (black circles), plantations with remnant rainforest trees (grey circles), and plantations without remnant rainforest trees (white circles), as well as the correlation of the ordination axis with important habitat parameters. Axis I clearly differentiates between the three habitat categories based on habitat quality, whereas the variation along axis 2 remains largely unexplained by the studied parameters.

unpublished data from M. Waltert, collected on Sulawesi during studies in 2001 and 2002. In some cases we had to use data from closely related species with similar body measurements. To assess whether birds feeding characteristics, stratum preferences, and degree of endemism differed between the habitat types, we used g-tests. To test whether bird body weight, representation of forest species versus generalists, and of endemics versus non-endemics differed between habitat type, we used ANOVAs.

\section{Results}

In total we observed 87 species in our 16 study plots (Table 2 ). Forty-one (48.3\%) of them were non-endemic resident species (henceforth called non-endemics), 41 (46\%) were endemics and $5(5.7 \%)$ were migrants. We found four species (Ficedula rufigula, Loriculus exilis, Ptilinopus subgularis and Zoothera erythronota) that are classified as 'Near Threatened' by IUCN (IUCN 2006) and 55 species that are of potential conservation concern being either endemic to the island or forest-dependent (Table 3 ).

The NMDS-analysis based on bird abundances grouped the plots into three categories (stress 12.3): near-primary forest (4 plots), plantations with remnant rainforest trees (7 plots), and plantations without remnant rainforest trees ( 5 plots) (Figure 1). Plantations differed significantly from forests in elevation (one-way ANOVA, $F_{2,13}=10.12, P<0.001$ ), canopy cover (one-way 
ANOVA, $F_{2,13}=12.58, P=0.001$ ), number of rainforest tree individuals (one-way ANOVA, $F_{2,13}=37.22, P<0.001$ ), and number of planted cacao trees (one-way ANOVA, $F_{2,13}=5.60$, $P=0.02)$. The two plantation types differed only in the number of remnant rainforest tree individuals (one-way ANOVA, $F_{2,13}=4.47, P=0.03$ ).

There was no significant difference in absolute bird species number between the three habitat types (g-test, $G$ (forest/plantations with remnant rainforest trees) $=0.2, G$ (forest/plantations without remnant rainforest trees) $=1.68, G$ (plantations without remnant rainforest trees/ plantations with remnant rainforest trees $)=0.73, P>0.05$ in all cases). Together, the four forest plots contained 65 species ( 29 non-endemics, 33 endemics, 3 migrants), plantations with remnant rainforest trees 60 ( 30 non-endemics, 27 endemics, 3 migrants), and plantations without remnant rainforest trees 51 ( 30 non-endemics, 19 endemics, 2 migrants). Near-primary forest had significantly more endemic species than plantations without remnant rainforest trees (g-test, $G=3.85, P<0.05$ ) but not significantly more than plantations with remnant rainforest trees $(G=0.80, P>0.05)$ and there was no significant difference between the two plantation types $(G=1.25, P>0.05)$.

Sörensen indices describing the similarity of species composition of all species, the endemics, non-endemics, generalists, forest specialists, the frugivores and the ground dwelling insectivores between the three different habitat types are given in Table 1 .

We classified $4 \mathrm{I}$ species as forest specialists (e.g., Meropogon forsteni, Heinrichia calligyna, Ptilinopus fischeri) and 46 as generalists (e.g., Dicaeum celebicum, Nectarinia jugularis, Lonchura molucca). We found 35 forest specialists in the forest plots, 21 in plantations with remnant rainforest trees and 12 in plantations without remnant rainforest trees. The forest plots had significantly more forest specialists than plantations (g-test, $G$ (plantations without remnant rainforest trees) $=19.52, P<0.01 ; G$ (plantations with remnant rainforest trees) $=8.62$, $P<0.01$ ). The two plantation types differed in the number of forest specialists but this was not significant. Among the forest specialists, we detected a significantly higher number of endemics and a lower number of generalists than in the entire data set (g-test, $G=5.03, P<0.05$ ).

Considering feeding guilds, we only found significantly more granivorous species in plantations without remnant rainforest trees than in the forest plots (g-test, $G=6.81, P<0.05$ ) but not more than in the plantations with remnant rainforest trees. All other feeding guilds were not significantly more common in any of the habitat types.

Comparing forests and plantations without remnant rainforest trees we found significantly more bird species dwelling mainly in the midstorey and canopy in the forest than in plantations without remnant rainforest trees (g-test, $G=23.32, P<0.01$ ). Plantations with remnant rainforest trees in comparison to forest showed significantly more generalist bird species not preferring one special stratum (g-test, $G=6.02, P<0.05$ ). Birds preferring other strata were not significantly more common in one of the habitat types. More generalist than forest specialist

Table 1 . Sörensen similarity indices of the community comparison of different groups of birds between the three habitat types; plantations with remnant rainforest trees $(\mathrm{ptI})$, plantations with remnant rainforest trees (ptII).

\begin{tabular}{llll}
\hline Similarity of: & $\begin{array}{l}\text { forest \& } \\
\text { ptI }\end{array}$ & $\begin{array}{l}\text { forest \& } \\
\text { ptII }\end{array}$ & $\begin{array}{l}\text { ptI \& } \\
\text { ptII }\end{array}$ \\
\hline all species & 65.5 & 70.4 & 73.9 \\
endemics & 65.4 & 73.3 & 65.2 \\
non-endemics & 65.6 & 67.7 & 80.0 \\
forest specialists & 46.8 & 57.1 & 42.4 \\
generalists & 78.3 & 81.2 & 87.2 \\
frugivores & 68.6 & 90.0 & 71.0 \\
ground dwelling insectivores & 66.7 & 72.7 & 86.7 \\
\hline
\end{tabular}


species were found in all strata, but this was not statistically significant (g-test, $G=3.58$, $P>0.05$ ). Forest specialists were significantly more abundant in the midstorey and canopy (g-test, $G=4.96, P<0.05$ ). Combining diet and stratum preference, we did not detect significant differences in the patterns of the three different habitat types. For example, there were not significantly more ground dwelling insectivores in forests than in one of the two plantation types.

Endemic species were not significantly heavier than the other species. Comparing the body weight of all species occurring in three habitat types likewise revealed no significant differences. However, forest species were significantly heavier than generalists (two-tailed ANOVA, $F_{1,86}=9.896 ; P=0.002$ ).

\section{Discussion}

In our study region, bird species diversity declined moderately from forest to plantations (Figure 2). The number of endemics showed a parallel decline. Fifteen species ( $20 \%$ of all species) were only recorded in natural forests and 41 species $(47 \%)$ were classified as forest specialists because they were mostly recorded in forests. No fewer than $61.9 \%$ of the forest specialists were endemics, whereas among the generalists this proportion was only $31.1 \%$. A further 14 endemic generalist species, most of them occurring in all habitat types, had to be regarded as being of potential conservation concern because they are restricted to Sulawesi and some are threatened (IUCN 2006). Thus, $56(63 \%)$ of the 87 species recorded by us, including 40 endemics, may be considered dependent on the presence of natural forests for their continued survival, although 40 of them were also found in plantations.

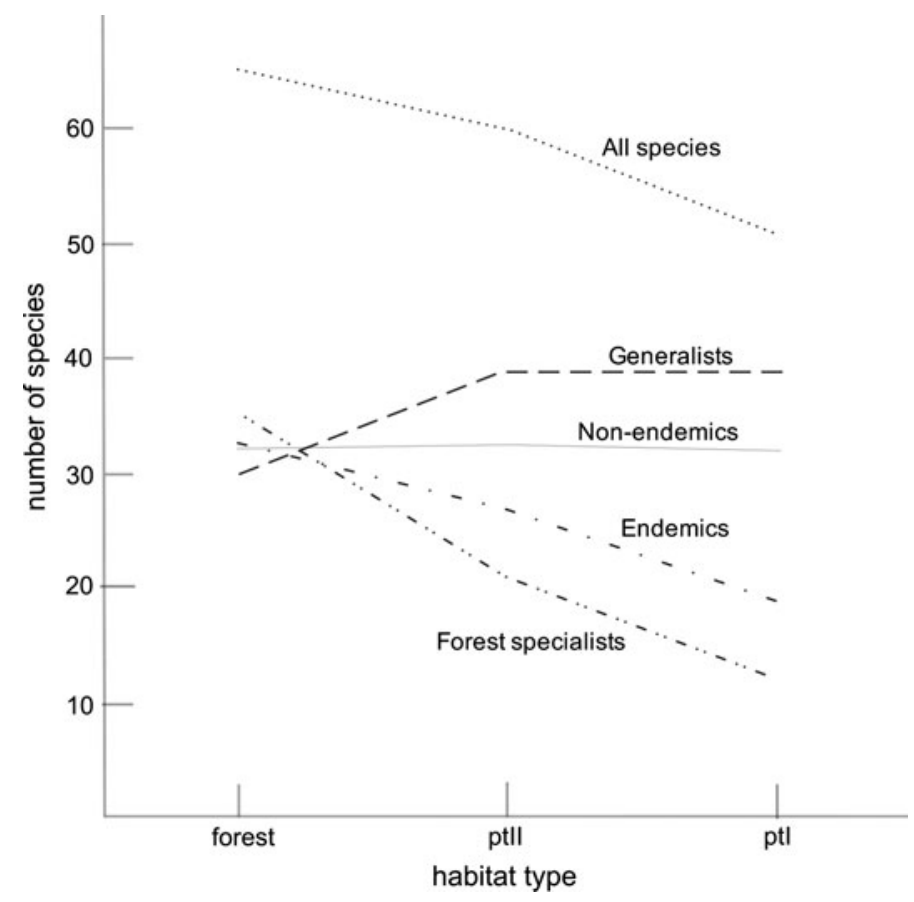

Figure 2. Number of bird species in the three habitat types (forest = near-primary forest, $\mathrm{ptII}=$ plantations with remnant forest trees, $\mathrm{ptI}=$ plantations without remnant trees). 
Table 2. Species characteristics and distribution (Distribution: $\mathrm{E}=$ endemic, $\mathrm{R}=$ resident, $\mathrm{V}=$ vagrant; Diet: $\mathrm{C}=$ carnivores, $\mathrm{F}=$ frugivores, $\mathrm{G}=$ granivores, $\mathrm{I}=$ insectivores, $\mathrm{N}=$ nectarivores, $\mathrm{O}=$ omnivores; Weight: $\mathrm{I}=\mathrm{o}-1 \mathrm{og}, 2=11-20 \mathrm{~g}, 3=21-40 \mathrm{~g}, 4=41-80 \mathrm{~g}, 5=81-160 \mathrm{~g}, 6=161-320 \mathrm{~g}, 7=321-640 \mathrm{~g}, 8=641-1280 \mathrm{~g}$, $9=>_{1280}$ g; Stratum $\mathrm{G}=$ ground $\&$ understorey, $\mathrm{M}=$ midstorey, $\mathrm{C}=$ canopy; Habitat: $\mathrm{F}=$ forest species, $\mathrm{G}=$ generalist).

\begin{tabular}{|c|c|c|c|c|c|c|c|c|}
\hline & Distribution & Diet & Weight & Stratum & Habitat & Forest & ptI & ptII \\
\hline Accipiter trinotatus & $\mathrm{E}$ & $\mathrm{C}$ & 5 & $\mathrm{M}$ & $\mathrm{F}$ & $\mathrm{x}$ & & $\mathrm{x}$ \\
\hline Aethopyga siparaja & $\mathrm{R}$ & $\mathrm{N}$ & 1 & GMC & G & $\mathrm{x}$ & $\mathrm{x}$ & $\mathrm{x}$ \\
\hline Anthreptes malacensis & $\mathrm{R}$ & $\mathrm{N}$ & 2 & $\mathrm{MC}$ & G & $\mathrm{x}$ & $\mathrm{x}$ & $\mathrm{x}$ \\
\hline Aplonis minor & $\mathrm{R}$ & $\mathrm{F}$ & 4 & $\mathrm{MC}$ & $\mathrm{F}$ & $\mathrm{x}$ & $\mathrm{x}$ & $\mathrm{x}$ \\
\hline Artamus monachus & $\mathrm{E}$ & I & 3 & $\mathrm{MC}$ & $\mathrm{F}$ & & & $\mathrm{x}$ \\
\hline Basilornis celebensis & $\mathrm{E}$ & $\mathrm{F}$ & 5 & $\mathrm{MC}$ & $\mathrm{F}$ & $\mathrm{x}$ & & $\mathrm{x}$ \\
\hline Bradypterus castaneus & $\mathrm{R}$ & I & 2 & G & $\mathrm{F}$ & & & $\mathrm{x}$ \\
\hline Cacomantis merulinus & $\mathrm{R}$ & I & 3 & $\mathrm{C}$ & G & $\mathrm{x}$ & $\mathrm{x}$ & $\mathrm{x}$ \\
\hline Cacomantis sepulcralis & $\mathrm{R}$ & I & 3 & GMC & G & $\mathrm{x}$ & $\mathrm{x}$ & $\mathrm{x}$ \\
\hline Caprimulgus celebensis & E & I & 4 & G & $\mathrm{F}$ & $\mathrm{x}$ & & \\
\hline Centropus bengalensis & $\mathrm{R}$ & I & 5 & GM & G & $\mathrm{x}$ & $\mathrm{x}$ & $\mathrm{x}$ \\
\hline Centropus celebensis & $\mathrm{E}$ & I & 7 & GMC & G & $\mathrm{x}$ & $\mathrm{x}$ & $\mathrm{x}$ \\
\hline Chrysococcyx russatus & $\mathrm{R}$ & I & 2 & GM & G & $\mathrm{x}$ & $\mathrm{x}$ & $\mathrm{x}$ \\
\hline Cisticola juncidis & $\mathrm{R}$ & I & 1 & G & G & & $\mathrm{x}$ & \\
\hline Coracina morio & $\mathrm{E}$ & I & 4 & $\mathrm{MC}$ & $\mathrm{F}$ & $\mathrm{x}$ & & $\mathrm{x}$ \\
\hline Coracina temminckii & $\mathrm{E}$ & I & 5 & $\mathrm{MC}$ & $\mathrm{F}$ & $\mathrm{x}$ & & \\
\hline Corvus enca & $\mathrm{R}$ & $\mathrm{O}$ & 7 & GMC & G & & $\mathrm{x}$ & $\mathrm{x}$ \\
\hline Cuculus crassirostris & $\mathrm{E}$ & I & 5 & $\mathrm{M}$ & G & $\mathrm{x}$ & $\mathrm{x}$ & $\mathrm{x}$ \\
\hline Cuculus saturatus & $\mathrm{V}$ & I & 5 & $\mathrm{MC}$ & $\mathrm{F}$ & $\mathrm{x}$ & & $\mathrm{x}$ \\
\hline Culicicapa helianthea & $\mathrm{R}$ & I & 1 & $\mathrm{MC}$ & G & $\mathrm{x}$ & $\mathrm{x}$ & $\mathrm{x}$ \\
\hline Cyornis omissus & $\mathrm{E}$ & I & 2 & GM & $\mathrm{F}$ & $\mathrm{x}$ & $\mathrm{x}$ & $\mathrm{x}$ \\
\hline Dendrocopos temminckii & $\mathrm{E}$ & I & 3 & $\mathrm{MC}$ & G & $\mathrm{x}$ & $\mathrm{x}$ & $\mathrm{x}$ \\
\hline Dicaeum aureolimbatum & $\mathrm{E}$ & $\mathrm{F}$ & 1 & GM & G & $\mathrm{x}$ & $\mathrm{x}$ & $\mathrm{x}$ \\
\hline Dicaeum celebicum & $\mathrm{E}$ & $\mathrm{F}$ & 1 & $\mathrm{C}$ & G & $\mathrm{x}$ & $\mathrm{x}$ & $\mathrm{x}$ \\
\hline Dicaeum nehrkorni & $\mathrm{E}$ & $\mathrm{F}$ & 1 & $\mathrm{C}$ & G & $\mathrm{x}$ & $\mathrm{x}$ & $\mathrm{x}$ \\
\hline Dicrurus hottentottus & $\mathrm{R}$ & $\mathrm{I}$ & 4 & $\mathrm{MC}$ & G & $\mathrm{x}$ & $\mathrm{x}$ & $\mathrm{x}$ \\
\hline Ducula forsteni & $\mathrm{E}$ & $\mathrm{F}$ & 7 & $\mathrm{MC}$ & G & $\mathrm{x}$ & $\mathrm{x}$ & $\mathrm{x}$ \\
\hline Enodes erythrophris & E & $\mathrm{F}$ & 5 & $\mathrm{MC}$ & $\mathrm{F}$ & $\mathrm{x}$ & & \\
\hline Eudynamys melanorhynchus & $\mathrm{R}$ & $\mathrm{F}$ & 6 & $\mathrm{MC}$ & $\mathrm{F}$ & $\mathrm{x}$ & & $\mathrm{x}$ \\
\hline Eurostopodus macrotis & $\mathrm{R}$ & $\mathrm{I}$ & 5 & G & G & $\mathrm{x}$ & $\mathrm{x}$ & $\mathrm{x}$ \\
\hline Falco moluccensis & $\mathrm{R}$ & $\mathrm{C}$ & 6 & G & G & & & $\mathrm{x}$ \\
\hline Ficedula hyperythra & $\mathrm{R}$ & I & 1 & G & $\mathrm{F}$ & $\mathrm{x}$ & & \\
\hline Ficedula rufigula & E & I & 2 & G & $\mathrm{F}$ & & $\mathrm{x}$ & \\
\hline Gallicolumba tristigmata & $\mathrm{E}$ & $\mathrm{F}$ & 6 & G & $\mathrm{F}$ & $\mathrm{x}$ & & \\
\hline Gallirallus philippensis & $\mathrm{R}$ & $\mathrm{O}$ & 6 & G & G & & $\mathrm{x}$ & $\mathrm{x}$ \\
\hline Gallus gallus & $\mathrm{R}$ & $\mathrm{O}$ & 8 & G & $\mathrm{F}$ & $\mathrm{x}$ & & \\
\hline Gerygone sulphurea & $\mathrm{R}$ & $\mathrm{I}$ & 1 & $\mathrm{MC}$ & G & & $\mathrm{x}$ & $\mathrm{x}$ \\
\hline Halcyon chloris & $\mathrm{R}$ & $\mathrm{C}$ & 4 & G & G & $\mathrm{x}$ & $\mathrm{x}$ & $\mathrm{x}$ \\
\hline Halcyon coromanda & V & $\mathrm{C}$ & 4 & G & G & $\mathrm{x}$ & & \\
\hline Haliastur indus & $\mathrm{R}$ & $\mathrm{C}$ & 7 & G & G & $\mathrm{x}$ & & \\
\hline Heinrichia calligyna & $\mathrm{E}$ & I & 3 & G & $\mathrm{F}$ & $\mathrm{x}$ & & \\
\hline Hieraaetus kienerii & $\mathrm{R}$ & $\mathrm{C}$ & 8 & G & $\mathrm{F}$ & $\mathrm{x}$ & & \\
\hline Hypothymis azurea & $\mathrm{R}$ & I & 2 & GMC & G & $\mathrm{x}$ & $\mathrm{x}$ & $\mathrm{x}$ \\
\hline Lonchura malacca & $\mathrm{R}$ & G & 2 & G & G & & $\mathrm{x}$ & \\
\hline Lonchura molucca & $\mathrm{R}$ & G & 2 & G & G & & $\mathrm{x}$ & $\mathrm{x}$ \\
\hline Lonchura punctulata & $\mathrm{R}$ & G & 2 & G & G & & $\mathrm{x}$ & \\
\hline Loriculus exilis & $\mathrm{E}$ & $\mathrm{F}$ & 2 & $\mathrm{C}$ & G & $\mathrm{x}$ & $\mathrm{x}$ & $\mathrm{x}$ \\
\hline Loriculus stigmatus & $\mathrm{E}$ & $\mathrm{F}$ & 3 & $\mathrm{C}$ & $\mathrm{F}$ & $\mathrm{x}$ & & $\mathrm{x}$ \\
\hline
\end{tabular}


Table 2. Continued.

\begin{tabular}{|c|c|c|c|c|c|c|c|c|}
\hline & Distribution & Diet & Weight & Stratum & Habitat & Forest & ptI & ptII \\
\hline Macropygia amboinensis & $\mathrm{R}$ & $\mathrm{F}$ & 5 & GMC & $\mathrm{F}$ & $\mathrm{x}$ & $\mathrm{x}$ & $\mathrm{x}$ \\
\hline Megapodius cumingii & $\mathrm{R}$ & I & 7 & GM & $\mathrm{F}$ & $\mathrm{x}$ & & \\
\hline Meropogon forsteni & $\mathrm{E}$ & I & 4 & $\mathrm{MC}$ & $\mathrm{F}$ & $\mathrm{x}$ & & \\
\hline Motacilla cinerea & $\mathrm{V}$ & I & 6 & G & G & & $\mathrm{x}$ & $\mathrm{x}$ \\
\hline Mulleripicus fulvus & $\mathrm{E}$ & I & 6 & GMC & $\mathrm{F}$ & $\mathrm{x}$ & $\mathrm{x}$ & $\mathrm{x}$ \\
\hline Muscicapa griseisticta & $\mathrm{V}$ & I & 2 & GMC & G & & & $\mathrm{x}$ \\
\hline Myzomela sanguinolenta & $\mathrm{R}$ & $\mathrm{N}$ & 1 & $\mathrm{MC}$ & G & $\mathrm{x}$ & $\mathrm{x}$ & $\mathrm{x}$ \\
\hline Nectarinia aspasia & $\mathrm{R}$ & $\mathrm{N}$ & 1 & GMC & G & $\mathrm{x}$ & $\mathrm{x}$ & $\mathrm{x}$ \\
\hline Nectarinia jugularis & $\mathrm{R}$ & $\mathrm{N}$ & 1 & GMC & G & & $\mathrm{x}$ & $\mathrm{x}$ \\
\hline Ninox punctulata & $\mathrm{E}$ & $\mathrm{C}$ & 5 & G & G & & & $\mathrm{x}$ \\
\hline Ninox scutulata & $\mathrm{V}$ & $\mathrm{I}$ & 6 & G & $\mathrm{F}$ & $\mathrm{x}$ & $\mathrm{x}$ & \\
\hline Oriolus chinensis & $\mathrm{R}$ & I & 4 & $\mathrm{C}$ & G & $\mathrm{x}$ & $\mathrm{x}$ & $\mathrm{x}$ \\
\hline Otus manadensis & $\mathrm{E}$ & $\mathrm{C}$ & 5 & G & $\mathrm{F}$ & $\mathrm{x}$ & $\mathrm{x}$ & \\
\hline Pachycephala sulfuriventer & $\mathrm{E}$ & I & 3 & $\mathrm{M}$ & $\mathrm{F}$ & $\mathrm{x}$ & $\mathrm{x}$ & \\
\hline Penelopides exarhatus & $\mathrm{E}$ & $\mathrm{F}$ & 7 & $\mathrm{C}$ & $\mathrm{F}$ & $\mathrm{x}$ & & $\mathrm{x}$ \\
\hline Phaenicophaeus calyorhynchus & $\mathrm{E}$ & I & 6 & GMC & $\mathrm{F}$ & $\mathrm{x}$ & $\mathrm{x}$ & $\mathrm{x}$ \\
\hline Phylloscopus sarasinorum & $\mathrm{E}$ & I & 1 & $\mathrm{MC}$ & $\mathrm{F}$ & & & $\mathrm{x}$ \\
\hline Pitta erythrogaster & $\mathrm{R}$ & I & 4 & GM & $\mathrm{F}$ & $\mathrm{x}$ & & \\
\hline Prioniturus platurus & $\mathrm{E}$ & $\mathrm{F}$ & 5 & GMC & $\mathrm{F}$ & $\mathrm{x}$ & & $\mathrm{x}$ \\
\hline Ptilinopus fischeri & $\mathrm{E}$ & $\mathrm{F}$ & 6 & $\mathrm{MC}$ & $\mathrm{F}$ & $\mathrm{x}$ & & \\
\hline Ptilinopus melanospilus & $\mathrm{R}$ & $\mathrm{F}$ & 5 & $\mathrm{C}$ & $\mathrm{F}$ & $\mathrm{x}$ & $\mathrm{x}$ & $\mathrm{x}$ \\
\hline Ptilinopus subgularis & E & $\mathrm{F}$ & 5 & $\mathrm{MC}$ & G & $\mathrm{x}$ & $\mathrm{x}$ & $\mathrm{x}$ \\
\hline Pycnonotus aurigaster & $\mathrm{R}$ & $\mathrm{F}$ & 4 & GMC & G & & $\mathrm{x}$ & \\
\hline Rhipidura teysmanni & $E$ & I & 2 & MC & $\mathrm{F}$ & $\mathrm{x}$ & & \\
\hline Rhyticeros cassidix & $E$ & $\mathrm{~F}$ & 9 & $\mathrm{C}$ & $\mathrm{F}$ & $\mathrm{x}$ & $\mathrm{x}$ & $\mathrm{x}$ \\
\hline Scissirostrum dubium & $E$ & $\mathrm{~F}$ & 4 & $\mathrm{C}$ & G & $\mathrm{x}$ & $\mathrm{x}$ & $\mathrm{x}$ \\
\hline Spilornis rufipectus & $E$ & $\mathrm{C}$ & 8 & G & $\mathrm{F}$ & & & $\mathrm{x}$ \\
\hline Spizaetus lanceolatus & $\mathrm{E}$ & $\mathrm{C}$ & 8 & $\mathrm{C}$ & $\mathrm{F}$ & & & $\mathrm{x}$ \\
\hline Streptocitta albicollis & $E$ & $\mathrm{~F}$ & 4 & $\mathrm{C}$ & G & $\mathrm{x}$ & & $\mathrm{x}$ \\
\hline Streptopelia chinensis & $\mathrm{R}$ & G & 5 & G & G & $\mathrm{x}$ & $\mathrm{x}$ & $\mathrm{x}$ \\
\hline Surniculus lugubris & $\mathrm{R}$ & I & 3 & $\mathrm{MC}$ & $\mathrm{F}$ & $\mathrm{x}$ & & \\
\hline Tanygnathus sumatranus & $\mathrm{R}$ & $\mathrm{F}$ & 6 & GMC & $\mathrm{F}$ & $\mathrm{x}$ & & $\mathrm{x}$ \\
\hline Treron vernans & $\mathrm{R}$ & $\mathrm{F}$ & 5 & $\mathrm{C}$ & $\mathrm{F}$ & $\mathrm{x}$ & $\mathrm{x}$ & \\
\hline Trichastoma celebense & $E$ & I & 3 & G & G & $\mathrm{x}$ & $\mathrm{x}$ & $\mathrm{x}$ \\
\hline Turnix chinensis & $\mathrm{R}$ & G & 3 & G & G & & & $\mathrm{x}$ \\
\hline Tyto rosenbergii & $\mathrm{E}$ & $\mathrm{C}$ & 8 & G & G & & $\mathrm{x}$ & \\
\hline Zoothera erythronota & $\mathrm{E}$ & I & 4 & G & $\mathrm{F}$ & $\mathrm{x}$ & & \\
\hline Zosterops atrifrons & $\mathrm{R}$ & I & 2 & GMC & G & $\mathrm{x}$ & $\mathrm{x}$ & $\mathrm{x}$ \\
\hline Zosterops chloris & $\mathrm{R}$ & I & 2 & GMC & G & & $\mathrm{x}$ & $\mathrm{x}$ \\
\hline
\end{tabular}

The change in habitat quality from the forest to the plantations was demonstrated distinctly by the decline of forest specialists. Plantations with a large number of rainforest trees harboured considerably more forest specialists (21) than plantations without rainforest trees (12). In contrast, the number of non-endemics remained constant but the similarity index of only about $50 \%$ revealed marked changes in the assemblage composition between forests and plantations. The number of generalists even increased slightly from forest to plantations and was constant within plantations (Figure 2). However, in contrast to the non-endemics, the similarity indices for generalists between the three habitat types were relatively high at around $80 \%$. Finally, the similarity in species composition between forests and plantations containing rainforest trees was much higher than between forests and plantations lacking rainforest trees (Table 1 ).

These overall patterns are typical of a comparison of forest habitats with agroforests, as found in many studies worldwide (e.g., Thiollay 1995, Greenberg et al. 1997, 2000, Fjeldså 1999, 
Table 3. Forest specialists, generalists and endemics (bold) occurring in the three habitat types; near-threatened species marked by asterisks.

\begin{tabular}{|c|c|c|c|c|}
\hline $\begin{array}{l}\text { forest specialists not } \\
\text { found in plantations }\end{array}$ & $\begin{array}{l}\text { forest specialists also } \\
\text { found in ptII }\end{array}$ & $\begin{array}{l}\text { forest specialists also } \\
\text { found in ptI }\end{array}$ & endemic generalists & non-endemic generalists \\
\hline $\begin{array}{l}\text { Caprimulgus celebensis } \\
\text { Coracina temminckii } \\
\text { Enodes erythrophris } \\
\text { Ficedula hyperythra } \\
\text { Gallicolumba tristigmata } \\
\text { Gallus gallus } \\
\text { Heinrichia calligyna } \\
\text { Hieraaetus kienerii } \\
\text { Megapodius cumingii } \\
\text { Meropogon forsteni } \\
\text { Pitta erythrogaster } \\
\text { Ptilinopus fischeri } \\
\text { Rhipidura teysmanni } \\
\text { Surniculus lugubris } \\
\text { Zoothera erythronota* }\end{array}$ & $\begin{array}{l}\text { Accipiter trinotatus } \\
\text { Aplonis minor } \\
\text { Artamus monachus } \\
\text { Basilornis celebensis } \\
\text { Bradypterus castaneus } \\
\text { Coracina morio } \\
\text { Cuculus saturatus } \\
\text { Cyornis omissus } \\
\text { Eudynamys melanorhynchus } \\
\text { Loriculus stigmatus } \\
\text { Macropygia amboinensis } \\
\text { Mulleripicus fulvus } \\
\text { Penelopides exarhatus } \\
\text { Phaenicophaeus calyorhynchus } \\
\text { Phylloscopus sarasinorum } \\
\text { Prioniturus platurus } \\
\text { Ptilinopus melanospilus } \\
\text { Rhyticeros cassidix } \\
\text { Spilornis rufipectus } \\
\text { Spizaetus lanceolatus } \\
\text { Tanygnathus sumatranus }\end{array}$ & $\begin{array}{l}\text { Aplonis minor } \\
\text { Cyornis omissus } \\
\text { Ficedula rufigula* } \\
\text { Macropygia amboinensis } \\
\text { Mulleripicus fulvus } \\
\text { Ninox scutulata } \\
\text { Otus manadensis } \\
\text { Pachycephala sulfuriventer } \\
\text { Phaenicophaeus calyorhynchus } \\
\text { Ptilinopus melanospilus } \\
\text { Rhyticeros cassidix } \\
\text { Treron vernans }\end{array}$ & $\begin{array}{l}\text { Centropus celebensis } \\
\text { Cuculus crassirostris } \\
\text { Dendrocopos temminckii } \\
\text { Dicaeum aureolimbatum } \\
\text { Dicaeum celebicum } \\
\text { Dicaeum nehrkorni } \\
\text { Ducula forsteni } \\
\text { Loriculus exilis* } \\
\text { Ninox punctulata } \\
\text { Ptilinopus subgularis* } \\
\text { Scissirostrum dubium } \\
\text { Streptocitta albicollis } \\
\text { Trichastoma celebense } \\
\text { Tyto rosenbergii }\end{array}$ & $\begin{array}{l}\text { Aethopyga siparaja } \\
\text { Anthreptes malacensis } \\
\text { Cacomantis merulinus } \\
\text { Cacomantis sepulcralis } \\
\text { Centropus bengalensis } \\
\text { Chrysococcyx russatus } \\
\text { Cisticola juncidis } \\
\text { Corvus enca } \\
\text { Culicicapa helianthea } \\
\text { Dicrurus hottentottus } \\
\text { Eurostopodus macrotis } \\
\text { Falco moluccensis } \\
\text { Gallirallus philippensis } \\
\text { Gerygone sulphurea } \\
\text { Halcyon chloris } \\
\text { Halcyon coromanda } \\
\text { Haliastur indus } \\
\text { Hypothymis azurea } \\
\text { Lonchura malacca } \\
\text { Lonchura molucca } \\
\text { Lonchura punctulata } \\
\text { Motacilla cinerea } \\
\text { Muscicapa griseisticta } \\
\text { Myzomela sanguinolenta } \\
\text { Nectarinia aspasia } \\
\text { Nectarinia jugularis } \\
\text { Oriolus chinensis } \\
\text { Pycnonotus aurigaster } \\
\text { Streptopelia chinensis } \\
\text { Turnix chinensis } \\
\text { Zosterops atrifrons } \\
\text { Zosterops chloris }\end{array}$ \\
\hline
\end{tabular}


Renjifo 2001, Schulze et al. 2004, Waltert et al. 2004a, Van Bael et al. 2007). These studies all show that bird diversity and community composition depend largely on habitat quality which in turn is mainly determined by the number of original rainforest trees providing a wide range of breeding niches and food resources for many species (Sodhi et al. 2005, Van Bael et al. 2007). Thus, the change in habitat quality is most pronounced between forest and plantations. The relatively low similarity of forest specialists (around 50\%) between forests and plantations and the 15 species only occurring in forests (forest specialists) show this clearly. Among the 15 forest specialists, including 10 endemics, that only occurred in forests, we found a high number of ecological specialists. Ten species are insectivorous and most of them are confined to one forest stratum, either to the ground or to the midstorey and canopy. These species, e.g., Meropogon forsteni or Coracina temminckii, need primary forest with its high habitat quality providing wellstructured strata and a great variability of food resources, and are of high conservation concern.

The relatively high number of forest specialists in the plantations compared to other studies (e.g., Greenberg et al. 2000, Harvey et al. 2006) has different reasons. First, the maximum distance of $300 \mathrm{~m}$ from the plantations to the forest border allowed birds to move into the plantations and back to the forest. Accordingly, the distance to the forest margin did not have a strong effect, as found in other studies elsewhere in the tropics (Reitsma et al. 2001, Faria et al. 2006). Second, the relatively low fragmentation in the lowland forests at the western border of Lore Lindu National Park (Waltert et al. 2004b) allows large forest specialists such as Spizaetus lanceolatus, Megapodius cumingii or Rhyticeros cassidix, that need extensive forest areas because they have on average larger territories than smaller species, to persist in the area. Third, due to the relatively high abundance of fruiting trees, we found a high number of typical forest frugivores such as Penelopides exarhatus, Tanygnathus sumatranus or Prinoturus platurus (Walker 2007) in plantations with remnant rainforest trees.

Plantations without rainforest trees had a smaller number of forest specialists than plantations with remnant rainforest trees and the similarity in species composition to the forest in all categories was lower than in plantations with remnant rainforest trees (Table 1 ). Structurally, the two plantation types differed from each other by the lack of original rainforest trees and an increasing number of fast growing, exotic tree species such as Gliricidia sepium, a small species from South America. This shows again that bird diversity and community composition depended largely on habitat quality. However, the presence of 12 forest specialists and the high number of generalists, including many endemics and the two near-threatened species Loriculus exilis and Ptilinopus subgularis, show that even plantations without rainforest trees have a certain value for bird conservation. Surely one reason for this is again the short distance to the forest and the low degree of disturbance within the forest, but the character of the plantations seems to be the most important point.

Contrary to other studies (e.g., Waltert et al. 2005a, b, Harvey et al. 2006, Faria et al. 2007, Van Bael et al. 2007), the number of ground dwelling insectivorous species was not significantly lower in plantations than in primary forest and the similarity in species composition was moderate to high $(66.7-72.7 \%)$. This is probably a consequence of the very low species diversity in understorey insectivores on Sulawesi in general (e.g., only one babbler and no insectivorous bulbul) and hence a broader realised niche of Sulawesi understorey insectivores compared to their congeners living more species-rich communities (such as, e.g., on Borneo). It is also possible that the density of birds in remaining forest habitats is not in equilibrium yet and that many individuals are 'trapped' in suboptimal conditions (Waltert et al. 2005b) or that at least for some species plantations are a sink habitat and that the bird species do not reproduce there (Sodhi et al. 2005). Also, the relatively high number of endemics occurring in the plantations can be explained in this way. Studies on this topic are sorely missing. For Sulawesi, in experiments with artificial nests, Pangau-Adam et al. (2006) found differences in egg predation rates in different types of forest, but the applicability of these results to our habitat types remains open.

Since only a few studies on bird diversity in forests and agroforest systems have been conducted on Sulawesi (Siebert 2002, Waltert et al. 2004b, Sodhi et al. 2005), it is interesting to 
compare the respective results. Compared to the other studies, we found a relatively high number of species in our plots. Some species like Otus manadensis or Ninox scutulata were not recorded in the other studies because observation started after dawn. Other species like Tanygnathus sumatranus or Zoothera erythronota are more typical of lowland forests and were not found in the other studies restricted to elevations above $1,100 \mathrm{~m}$. In turn, these studies reported more typically montane taxa such as Dicrurus montanus or Myza celebensis not found by us. More intriguingly, some species that should have been found in all studies were missing in some. For example, Surniculus lugubris or Dicaeum nehrkornii were only recorded in our study. It is possible that these species may be unable to persist at their upper elevational ranges if the adjacent lowland areas have been deforested (Waltert et al. 2004a), explaining the absence of these species from the other study sites that were flanked from below by agricultural areas. The effect of deforestation in the lowland forests may also be perceivable in Toro valley were typical lowland species such as Cittura cyanotis, Actenoides monachus, Ceyx fallax, Corvus typicus, and Trichoglossus flavioidis were surprisingly missing although at other sites they occur at the elevation of Toro valley. All of these species were also missed by Siebert (2002) and Sodhi et al. (2005). Except for Ceyx fallax (Waltert et al. 2005b) these species do not occur in the plantations, suggesting that they depend on unfragmented lowland forest. The ongoing deforestation of this, the most severely fragmented habitat type in the study region, is a serious threat to the existence of these species.

Three main conclusions can be drawn from our study. First, the most critically threatened taxa are those occurring primarily at elevations below $1,000-1,200 \mathrm{~m}$. Conservation of these species in Lore Lindu National Park and other reserves in Sulawesi facing deforestation in their lower parts will depend on the stabilisation of the current natural forest boundary, securing adequate areas of forest at low elevations (Waltert et al. 2004b). Second, we found that structurally complex plantations on the margins of Lore Lindu National Park can provide suitable habitats especially for habitat generalists and some forest specialists. However, the present plantation management with an increasing elimination of natural shade trees as well as ongoing deforestation and forest fragmentation, will result in the loss of most forest specialists from the plantations. Accordingly, management schemes should aim to maintain a high density and diversity of shade trees in agroforests. This has been successfully implemented in coffee plantations in Central America (certified shade-grown coffee) and is similarly economically feasible for cacao plantations (Steffan-Dewenter et al. 2007). Third, future studies should focus on the population biology (nesting success, longevity, dispersal, etc.) of selected species of conservation concern present in agroforests to assess the potential of these habitats in maintaining viable populations of these species.

\section{Acknowledgements}

This article originated from STORMA, the Collaborative Research Centre 552 funded by the German Research Council (Deutsche Forschungsgemeinschaft DFG). Special thanks go to Yann Clough for his help with the organization, Dr. Stefan Erasmi and his team for providing the GISdata, to Navjot S. Sodhi and his team for providing data of their ornithological study, and to two anonymous reviewers for valuable comments on an earlier version of the manuscript.

\section{References}

Achard, F., Eva, H., Stibig, H., Mayaux, P., Gallego, J., Richards, T. and Malingreau, J. (2002) Determination of deforestation rates of the worlds humid tropical forests. Science 297: 999-1002.
Bos, M., Steffan-Dewenter, I. and Tscharntke, T. (2007) The contribution of cacao agroforests to the conservation of lower canopy ant and beetle diversity in Indonesia. Biodivers. Conserv. 16: 2429-2444. 
Brook, B., Sodhi, N. and Ng, P. (2003) Catastrophic extinctions follow deforestation in Singapore. Nature 424: 420-423.

Bryant, D., Nielsen, D. and Tampley, L. (1997) The last frontier forest: Ecosystems and economics on the edge. Washington, DC: World Resources Institute.

Coates, B., Bishop, K. and Gardner, D. (1997) A guide to the birds of Wallacea: Sulawesi, the Moluccas and the Lesser Sunda Islands, Indonesia. Alderley, Queensland: Dove Publications.

Del Hoyo, J., Elliott, A. and Sargatal, J. Eds. (1992-2006) Handbook of the birds of the world, Volumes 1-11. Barcelona: Lynx Edicions.

Faria, D., Laps, R., Baumgarten, J. and Cetra, M. (2006) Bat and bird assemblages from forests and shade cacao plantations in two contrasting landscapes in the Atlantic forests of southern Bahia, Brazil. Biodivers. Conserv. 15: 587-612.

Faria, D., Paciencia, M., Dixo, M., Laps, R. and Baumgarten, J. (2007) Ferns, frogs, lizards, birds and bats in forest fragments and shade cacao plantations in two contrastino landscapes in the Atlantic forest, Brazil. Biodivers. Conserv. 16: 23352357.

Feare, C. and Craig, A. (1999) Starlings and mynas. Princeton, New Jersey: Princeton University Press.

Fjeldså, J. (1999) The impact of human forest disturbance on the endemic avifauna of the Udzungwa Mountains, Tanzania. Bird Conserv. Internatn. 9: 47-62.

Fox, J., Truong, D., Rambo, T., Tuyen, N., Cuc, L. and Leisz, S. (2000) Shifting cultivation: a new old paradigm for managing tropical forests. BioScience 50: 521-528.

Gradstein, S. R., Kessler, M. and Pitopang, R. (2007) Tree species diversity relative to human land uses in tropical rain forest margins in Central Sulawesi. Pp. 321-334 in: T. Tscharntke, C. Leuschner, M. Zeller, E. Guhardja and A. Bidin, eds. The stability of tropical rainforest margins: linking ecological, economic and social constraints of land uses and conservation. Berlin: Springer.

Greenberg, R., Bichier, P. and Sterling, J. (1997) Bird populations in shade and sun coffee plantations of eastern Chiapas, Mexico. Biotropica 29: 501-514.

Greenberg, R., Bichier, P. and Angón, A. (2000) The conservation value for birds of cacao plantations with diverse planted shade in Tabasco, Mexico. Anim. Conserv. 3: 105-112.

Harvey, C. A., Medina, A., Merlo Sánchez, D., Vílchez, S., Hernández, B., Sáenz, J. C., Maes, J. M., Casanoves, F. and Sinclair, F. L. (2006) Patterns of animal diversity associated with different forms of tree cover retained in agricultural landscapes. Ecol. Applic. 16: 1986-1999.

Higgins, P. and Peter, J. (2002) Handbook of Australian, New Zealand and Antarctic birds. Vol. 6: Pardalotes to shrike-thrushes. Melbourne: Oxford University Press.

Higgins, P., Peter, J. and Cowling, S. (2006) Handbook of Australian, New Zealand and Antarctic Birds. Vol. 7 (Dunnock to Starlings) Part B. Melbourne: Oxford University Press.

IUCN 2006. 2006 IUCN Red List of Threatened Species. Cambridge, UK: IUCN.

Maher, W. 1991: Growth and Development of Yellow-bellied sunbird Nectarinia jugularis in North Queensland. Emu 91: 58-61.

Myers, N., Mittermeier, R., Mittermeier, C., da Fonseca, G. and Kent, J. (2000) Biodiversity hotspots for conservation priorities. Nature 403: 853-858.

Pangau-Adam, M., Waltert, M. and Mühlenberg, M. (2006) Nest predation risk on ground and shrub nests in forest margin areas on Sulawesi, Indonesia. Biodivers. Conserv. 15: 4143-4158.

Parker, T. A. III. 1991: On the use of tape recorders in avifaunal surveys. Auk 108: 443-444.

Reitsma, R., Parrish, J. and McLarney, W. (2001) The role of cacao plantations in maintaining forest avian diversity in southeastern Costa Rica. Agroforest Syst. 53: 185-193.

Renjifo, L. (2001) Effect of natural and anthropogenic landscape matrices on the abundance of subAndean bird species. Ecol. Applic. 11: 14-31.

Rice, R. and Greenberg, R. (2000) Cacao cultivation and the conservation of biological diversity. Ambio 29: 167-173. 
Schroth, G., Harvey, C. and Vincent, G. (2004) Complex agroforests: their structure, diversity, and potential role in landscape conservation. Pp. $227-260$ in G. Schroth, G. Fonseca, C. Harvey, C. Gascon, H. Vasconcelos and A. Izac, eds. Agroforestry and conservation of biodiversity in tropical landscapes. Washington: Island Press.

Schulze, C., Waltert, M., Kessler, P., Pitopang, R., Shahabuddin, Veddeler, D., Mühlenberg, M., Gradstein, R., Leuschner, C., Steffan-Dewenter, I. and Tscharntke, T. (2004) Biodiversity indicator groups of tropical land-use systems: Comparing plants, bird, and insects. Ecol. Applic. 14: 1321-1333.

Siebert, S. (2002) From shade- to sun-grown perennial crops in Sulawesi, Indonesia: implications for biodiversity conservation and soil fertility. Biodivers. Conserv. I1: 1889-1902.

Sodhi, N., Pin Koh, L., Prawiradilaga, D., Idris Tinulele, D., Dwi Putra, D. and Han Tong Tan, T. (2005) Land use and conservation value for forest birds in Central Sulawesi. Biol. Conserv. 122: 547-558.

Stattersfield, A., Crosby, N., Long, A. and Wege, D. (1998) Endemic bird areas of the world. Priority areas for biodiversity conservation. Cambridge, UK: Birdlife International. Birdlife Conservation Series 7.

Steffan-Dewenter, I., Kessler, M., Barkmann, J., Bos, M., Buchori, D., Erasmi, S., Faust, H., Gerold, G., Glenk, K., Gradstein, R., Guhardja, E., Harteveld, M., Hertel, D., Höhn, P., Kappas, M., Köhler, S., Leuschner, C., Maertens, M., Marggraf, R., Migge-Kleian, S., Mogea, J., Pitopang, R., Schaefer, M., Schwarze, S., Sporn, S., Steingrebe, A., Tijtrosoedirdjo, S., Tijtrosoemito, S., Twele, A., Weber, R., Woltmann, L., Zeller, M. and Tscharntke, T. (2007) Tradeoffs between income, biodiver- sity, and ecosystem functioning during tropical rainforest conversion and agroforestry intensification. Proc. Natl. Acad. Sci. 104: 4973-4978.

The Nature Conservancy (2002) Lore Lindu National Park Draft Management Plan 2002-2027, vol. 1. Arlington, Virginia: The Nature Conservancy.

Thiollay, J. (1995) The role of traditional agroforests in the conservation of rain forest bird diversity in Sumatra. Conserv. Biol. 9: 335-353.

Van Bael, S., Bichier, P., Ochoa, I. and Greenberg, R. (2007) Bird diversity in cacao farms and fragments of western Panama. Biodivers. Conserv. 16: 2245-2256.

Walker, J. (2007) Dietary specialisation and fruit availability among frugivorous birds on Sulawesi. Ibis 10: 1-12.

Waltert, M., Mardiastuti, A. and Mühlenberg, M. (2004a) Effects of land use on bird species richness in Sulawesi, Indonesia. Conserv. Biol. 18: 1339-1346.

Waltert, M., Langkau, M., Fermon, H., Maertens, M., Härtel, M., Erasmi, S. and Mühlenberg, M. (2004b) Predicting losses of lowland bird species from deforestation in Central Sulawesi. Pp 327-350 in G. Gerold, M. Fremerey and E. Guhardja, eds. Land use, nature conservation and the stability of rainforest margins in Southeast Asia. Berlin: Springer.

Waltert, M., Bobo, K. S., Sainge, N. M., Fermon, H. and Mühlenberg, M. (2005a) From forest to farmland: habitat effects on Afrotropical forest bird diversity. Ecol. Applic. 15: 1351-1366.

Waltert, M., Mardiastudi, A., Mühlenberg, M. (2005b) Effects of deforestation and forest modification on understory birds in Central Sulawesi. Bird Conserv. Internatn. 15: 257-273. 
STEFAN ABRAHAMCZYK*, MICHAEL KESSLER

Albrecht-von-Haller-Institute of Plant Sciences, Georg-August University, Untere Karspüle 2, 37073 Göttingen, Germany.

DADANG DWI PUTRA

Celebes Bird Club, clo Balai Penelitian dan Pengembangan Zoologi, Puslitbang Biologi-LIPI, Jl. Raya Bogor Jakarta Km 46, Cibinong 16911, Indonesia.

\section{MATTHIAS WALTERT}

Department of Conservation Biology, Centre for Nature Conservation, Georg-August Universität, Von-Siebold-Str. 2, 37075 Göttingen, Germany.

TEJA TSCHARNTKE

Department of Crop Science, Agroecology, Georg-August-Universität, Waldweg 26, 37073 Göttingen, Germany.

${ }^{*}$ Author for correspondence.e-mail: sabraha@gwdg.de

Received 13 September 2007; revision accepted 11 February 2008 